\title{
A record system for contact tracing
}

\section{ANN SATIN \\ From St Thomas' Hospital and Health Education Council, London}

SUMMARY A system for recording information on patients and contacts was developed during a research project designed to measure the effectiveness of contact tracing. The record system has proved valuable in contact tracing, cross-referencing patients and their contacts, defining the characteristics of the patient and contact populations, and providing information for research and management. The value of a standardised system has been accepted by health workers who appreciate that its purpose is to increase efficiency and improve the care of infected persons. By October 1976 health workers in 16 clinics in the United Kingdom had started to use the system.

\section{Introduction}

Tracing, identifying, and locating contacts of patients with gonorrhoea and/or syphilis has for many years been considered an important part of disease control in the United Kingdom. However, little is known about the effectiveness of contact tracing or how to improve it. In 1971 the Health Education Council, in co-operation with the London boroughs of Lambeth and Wandsworth and the Sexually Transmitted Diseases (STDs) Clinic at St Thomas' Hospital, began a research project to investigate the effectiveness of contact tracing.

The effectiveness of contact tracing or any other health care endeavour can be assessed if objectives are defined and an information system is available from which details of the problem, action taken, and the results can be extracted and measured. At the start of the project it was discovered that few such details were available, and it was necessary to devise an information system to collect reliable data.

In designing an information system consideration was given to the details of contact tracing and the requirements of the persons who carry out this task.* The system was designed to record separately from the medical notes detailed information about patients and all the actions taken by the health worker in identifying and locating contacts.

The objective of contact tracing is to ensure that the individuals who have had sexual contact with infected patients seek medical examination and treatment when necessary. Therefore the information system was devised to record details about patients and their sexual contacts. A pilot study revealed that health workers used informal systems, recording

\footnotetext{
*Since 1974 those persons formerly known as contact tracers have preferred to be called health workers.

Address for reprints: 36 Milman Road, London NW6 Received for publication 20 October 1976
}

information about patients and contacts together in personal notebooks and in the medical notes. It was decided that the most useful and flexible system would be to provide separate documents: a card for each patient interviewed and a card for each contact identified. This simplified cross-referencing patients and contacts' details. For research it was useful to be able to separate patient and contact populations to examine their characteristics and the degree of overlap between them.

The pilot evaluation also showed that information about contacts could be better documented. The new concept of a record for each contact suspected of infection would provide more accurate and consistent information about the contacts and the size of a potentially infected population. Separate contact records would facilitate cross-reference to discover which contacts were named by more than one patient. Information kept separately from the medical files would protect the confidentiality of the non-medical details to be recorded.

Confidentiality of records of patients with STDs is a sensitive issue. Care is necessary in handling information about the private and intimate behaviour of the patient who has attended a clinic and about persons who are not present and perhaps unaware of the disclosures and their implications. The information system uses the safeguard of linking records only by numbers; the patient details are recorded separately from the contact details, and the information concerning numerous contacts of a single patient are also kept apart.

\section{Description of the structure and use of the system}

THE WHITE PATIENT CARD

Contact tracing is centred around the interview of a patient who has been diagnosed and treated. A patient card is completed for each patient interviewed for a new infection. Details of further inter- 
views with the same patient during treatment and after care are recorded on the same card. Should the patient return with a new infection a new card is made out. This provides a record of repeated infection and the separate contact details which are relevant to each new infection.

The information to be recorded is clearly labelled. The clinic number, the dates of attendance, and treatment, as well as details of contact tracing interview and the diagnosis are recorded along the top section (Fig. 1). The identification and descriptive details, personal, and civil data are recorded on the left side of the card. There is space to record anything about the history that might be useful in contact tracing. A section defining the source of attendance is included. These categories are not exclusive as it is possible that a patient may have attended as a result of a combination of factors.

The section labelled CONTACT INFORMATION is a summary of information about each of the sexual partners reported to be involved. The contact number is a unique cross-reference number which appears on the contact card. If a contact attends a clinic and is identified as a patient, space is provided to record, name, clinic number, and diagnosis.

The comments section is used to record reinterviews and other communication with and about the patient such as phone calls, letters, or home visits. There is space for the date of each interaction to be recorded. The back of the card gives additional space for comments and records of contact summaries when a patient names more than five partners.

\section{THE YELLOW CONTACT CARD}

A contact card (Fig. 2) is completed for each sexual partner who might be involved in an infection even if the person is probably untraceable, has already received treatment, or has been named by another patient. Health workers need to know enough about the infections to decide which individuals should be included in contact investigation. The card system provides a record of specific dates for this purpose.

The information along the top of the card, above the double line, relates to the patient and provides the cross-reference information-that is, the clinic number, the diagnosis, and the date that the contact

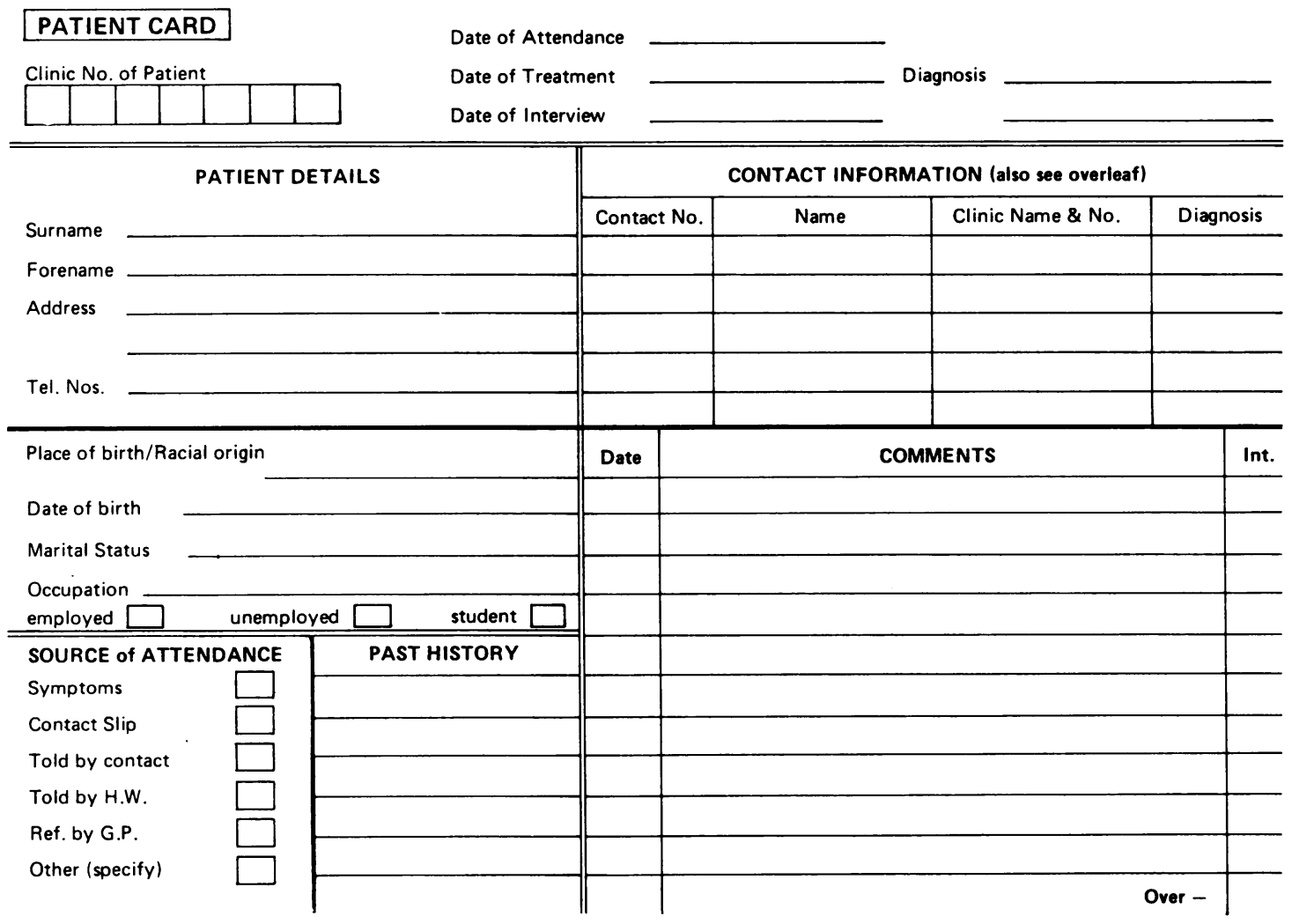

Fig. 1 The patient card 


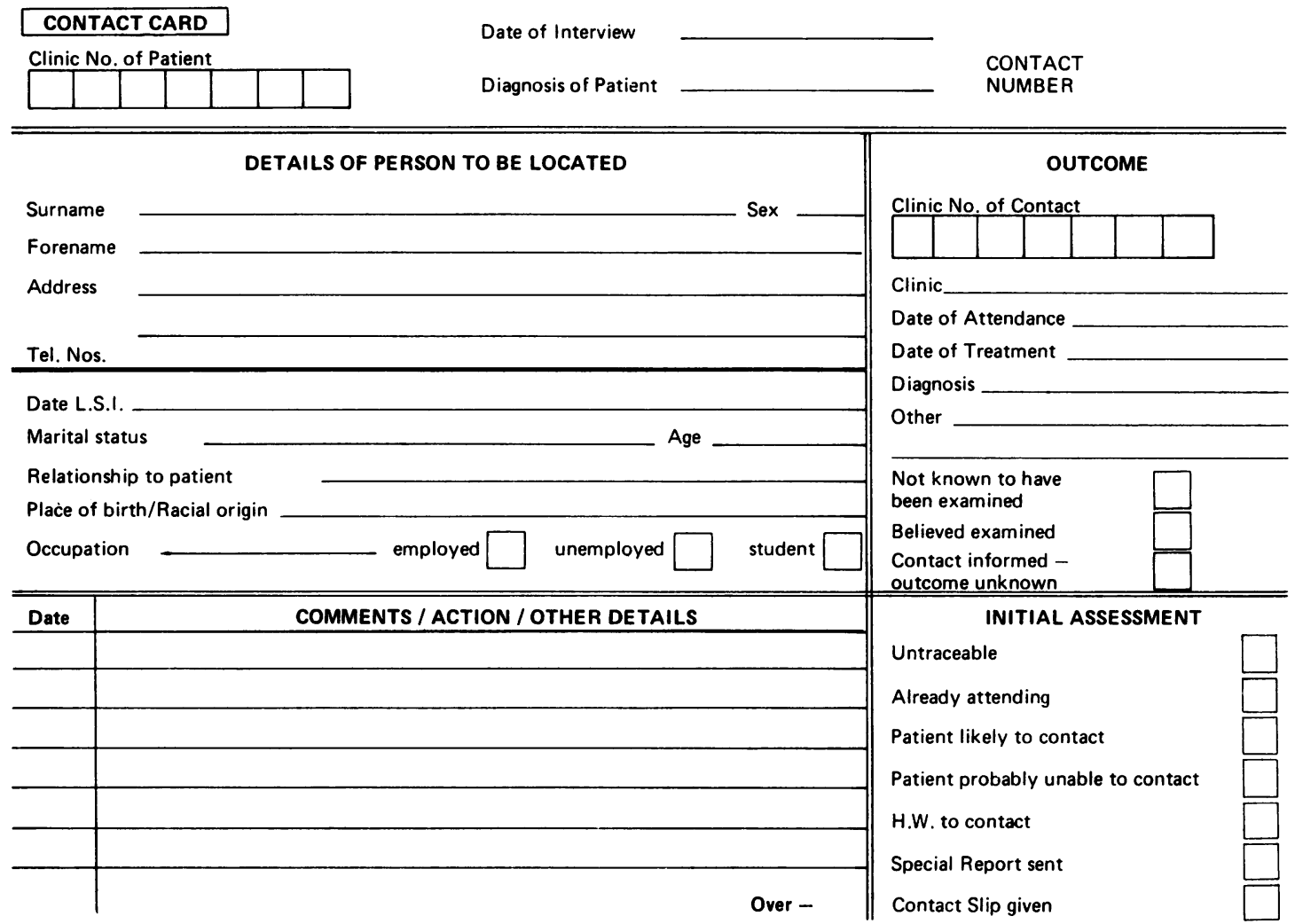

Fig. 2 The contact card

identifying information was given. The unique contact number is in the upper right corner. The cards are numbered sequentially, and it is this card number which is recorded as the contact number in the summary section of the patient card.

All information recorded below the double line concerns the contact. In addition to identifying and locating information, the date of the last sexual intercourse (LSI) is recorded. The space for COMMENTS/ACTION/OTHER DETAILS is for the contact's description, and it provides space for a history of the sequence of telephone calls, visits, and information exchanged with other clinics that are in progress or were undertaken to locate a contact.

At the first interview the interviewer assesses the likelihood of bringing a contact to examination, and what action the patient and/or the health worker may initiate to achieve this. This information is recorded in the section labelled INITIAL ASSESSMENT to guide subsequent action. The categories are not exclusive. It is here that the health worker indicates if a contact slip was issued.

The outсоме section is a record of the result of the investigation, and the details required are labelled. The back of the card is a continuation of the COMMENTS section.

THE CARD LAYOUT

In designing the cards both fixed and free formats were used. In general, fixed format sections improve recording and are easier to complete and analyse. The fixed response, however, must be accurate and in terms relevant to the user. Some terms which health workers and other staff use are ambiguous, particularly those used to classify sexual behaviour and the meaning and duration of relationships with sex partners. Therefore, information about sexual behaviour and partner relationships are not recorded in fixed categories such as 'casual', 'regular', 'active homosexual', 'passive homosexual', 'primary contact', 'secondary contact' because these rigid definitions were found to be unreliable. The free format was also found to be more appropriate for recording the health workers' activities.

FILING AND STORING CARDS

The recommended method for filing cards is that the 
patient cards are filed by patient number order, separated firstly by sex of patient, secondly by month of interview. The contact cards are initially filed with the corresponding patient cards until the investigation ends. When the outcome is known, contact cards are removed from the current file and filed apart in sequence according to card number; they are available for reference when necessary.

\section{Extension of the record system to other clinics}

After the pilot system was tested and found to be successful at St Thomas' Hospital its use was extended to selected UK clinics. The health workers who have taken up the system have made valuable assessments and suggestions for modifications to the cards which have been incorporated. Some health workers found it difficult to accept that they could all use the same system because they tended to see their particular situation as unique. None the less they felt that some structured system of recording information was desirable. Experienced health workers can use the system without difficulty using an instruction sheet for guidance. Health workers new to the work have reported that the information system has provided them with a secure structure for beginning the sensitive task of interviewing for contact information.

The system has been accepted readily by health workers who appreciate that its purpose is to increase efficiency and improve the care of infected persons. The value of standardised information for management and research purposes is also recognised. Since October 1976, health workers in 16 clinics, seven in London, nine outside, have used this system.

\section{The use of the system for research and management purposes}

Data collected from the system after the project was established at St Thomas' Hospital has been the basis for several descriptive studies* of the patient and contact populations. It is not within the scope of this paper to report the results of these studies or subsequent comparative studies which were carried out after the system had been introduced into other clinics. However, the information which has been or can be made available for analysis includes:

1. The number of patients interviewed by health workers, their sex and diagnosis, and other characteristics such as sexual orientation, employment status, occupation, nationality, and ethnic group.

\footnotetext{
*Individual reports are cited in The Control of the Spread of Gonorrhoea; A Health Education Exercise to Improve Contact Tracing 1975. Appendix 'A' page 17. Health Education Council.
}

2. The number of contacts named and their personal data in relation to patient characteristics.

3. The number of contacts named who were known or believed to have been examined, and their diagnoses.

4. The number of contacts named but not traced.

Of particular significance to the epidemiology of STD is the time interval between contraction and treatment of an infection. The system can also be used to collect the following information:

1. For patients, the amount of time which elapses between attendance at a clinic, establishment of diagnosis, treatment and interview, and dates of sexual intercourse with contacts.

2. For contacts, the amount of time which elapses between date of sexual intercourse, the contact being named by a patient and attendance at a clinic for examination.

The use of a structured system of recording information has implications apart from the increased reliability of the data that can result. The use of a standard system avoids duplication and facilitates continuity especially in large clinics with two or more health workers and when there is staff change-over. The system also aids communication between clinics as information is available in a standard format and is readily retrievable. The use of a standard system means that in the event of absence due to holidays, illness, or other unforeseen events health workers familiar with the system can substitute for one another. The system provides health workers with the means of assessing the progress and outcome of each contact investigation. This keeps the health worker aware of unfinished and unresolved cases that may require more effort.

The system can provide data for standard comparable measures of effectiveness. Studies have been undertaken which indicate several ways of assessing effectiveness. The results of these investigations are to be reported with the intention of stimulating discussion which will enhance the understanding of contact tracing as a health care activity.

The information system described was developed by a team from the former Medical Research Division of the Health Education Council whose members included Dr J. W. Dale, Rosemary Samways, Angela Mills, Sally Poole, Mary Ann Sawyer, Donald Simpson, and Hermine Whitfield. Dr C. S. Nicol provided support and encouragement. Health workers from many clinics have contributed by their criticism, co-operation, and enthusiasm. 